\title{
Dagli scranni accademici alle sdraio... sdraie... sdral???
}

\author{
Matilde Paoli
}

PUBBLICATO: 29 LUGLIO 2011

\section{Quesito:}

Ciclicamente, all'avvicinarsi dell'estate qualcuno, questa volta Guglielmo Tarditi da Torino, Diego Capozucca dalla provincia di Fermo, Michel Giovannini da Trieste, ci chiede quale sia la forma da usare in italiano per indicare la 'sedia a sdraio' e in particolare quale sia il plurale corretto.

\section{Dagli scranni accademici alle sdraio... sdraie... sdrai???}

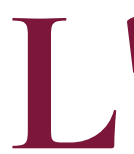

1 ingresso del sintagma sedia a sdraio in lingua viene "ufficializzato" nella prima metà dell'Ottocento dal Vocabolario della lingua italiana "già compilato dagli accademici della Crusca ed ora nuovamente accresciuto dall'abate Giuseppe Manuzzi" (I833), nel quale alla voce sdraio si legge: "'Seggiola' o 'poltrona a sdraio': dicesi quella seggiola o poltrona fatta in modo da starvi disteso comodamente".

Probabilmente l'abate Manuzzi parlava di un oggetto diverso da quella sedia pieghevole con seduta di stoffa che siamo abituati a vedere sulle spiagge e al bordo delle piscine durante l'estate e che ha cominciato a divenire popolare negli anni Sessanta del secolo scorso. Le prime attestazioni letterarie della riduzione del sintagma al solo sostantivo (da sedia a sdraio a sdraio) risalgono proprio a quegli anni, quando le vacanze al mare divennero uno dei primi "lussi" consentiti alla classe media del dopoguerra e l'uso della sedia a sdraio cominciò a diffondersi sui litorali della penisola.

Per alcuni autori, come Alessandro Bonsanti, nel romanzo I cavalli di bronzo pubblicato nel i956, e Edoardo Sanguineti, nel Giuoco dell'Oca del 1967, il sostantivo sdraio assume il genere della locuzione da cui deriva e resta invariabile al plurale; ed è così che viene registrato dalla maggior parte dei dizionari di lingua contemporanei.

In altri scrittori però, come Cesare Pavese (La Spiaggia, I959) e Vittorio Sereni (Gli strumenti umani, I969), il genere è determinato da sdraio, che esisteva (ed esiste tuttora) come sostantivo maschile per indicare 'lo sdraiarsi, lo star coricato' e, più recentemente, anche 'breve riposo, siesta'. Questo sostantivo ha la forma plurale gli sdrai.

Infine abbiamo anche l'adattamento morfologico sdraia che trova la prima attestazione letteraria in Palazzeschi (I fratelli Cuccoli, I948) ed è stato successivamente usato da Savinio, Pasolini e Montale, ma che solo GRADIT, tra i dizionari contemporanei, registra, glossandolo per di più come "di basso uso".

Da un sondaggio in rete emerge l'indiscutibile predominanza nell'uso attuale di sdraio femminile invariabile, ma si nota anche altrettanto chiaramente la concentrazione della forma maschile (lo sdraio, gli sdrai) in area nord-orientale (moltissime le attestazioni di operatori turistici della riviera romagnola) e la maggior diffusione di quella femminile numerabile (la sdraia, le sdraie) in area centromeridionale (molte attestazioni in dialetto romanesco o pseudo tale).

Lasciando da parte una possibile differenziazione in rapporto a usi regionali, possiamo dire che sedia/poltrona a sdraio, sdraio femminile invariabile e sdraia o sdraio maschile numerabile, si collocano in una progressione dal registro formale, a quello di media formalità, fino a quello decisamente familiare. 


\section{Cita come:}

Matilde Paoli, Dagli scranni accademici alle sdraio... sdraie... sdrai???, "Italiano digitale", 2011, X, 2019/3 (luglio-settembre)

DOI: $10.35948 / 2532-9006 / 2020.3208$

Copyright 2011 Accademia della Crusca

Pubblicato con licenza creative commons CC BY-NC-ND 